\title{
Life Cycle and Lensing of a Macular Microcyst
}

\author{
Alexander Meadway ${ }^{a} \quad$ Alex S. McKeown ${ }^{a} \quad$ Brian C. Samuels $^{b}$ \\ Lawrence C. Sincich ${ }^{\mathrm{a}}$ \\ a Department of Optometry and Vision Science, School of Optometry, University of Alabama at Birmingham, \\ Birmingham, AL, USA; ${ }^{b}$ Department of Ophthalmology and Visual Sciences, School of Medicine, University of \\ Alabama at Birmingham, Birmingham, AL, USA
}

\section{Keywords}

Adaptive optics · Photoreceptor mosaic · Optical modeling $\cdot$ Schisis

\begin{abstract}
Introduction: Microscopic details about retinal conditions can provide insight into pathological mechanisms, but these are ordinarily difficult to obtain in situ. We demonstrate how high-resolution imaging and optical modeling can be combined to reveal morphological features of a macular microcyst, offering insight into microcyst formation. Objective: To use adaptive optics scanning laser ophthalmoscopic (AOSLO) images to track a transient retinal microcyst and derive its 3-dimensional shape. Methods: A series of AOSLO images were gathered before, during, and after a transient retinal microcyst developed in an otherwise normal healthy 26-yearold male subject. Optical coherence tomography (OCT) independently confirmed the location of the microcyst. Optical modeling was conducted to quantify the lensing effect of the optically uniform microcyst and to determine its 3-dimensional shape. Increment threshold sensitivity, targeted within and around the microcyst, was tested to see if cone photoreceptor function was affected. Results: A transient microcyst appeared as a $50 \mu \mathrm{m}$ diameter circle in AOSLO im-
\end{abstract}

karger@karger.com www.karger.com/ore

(c) 2020 S. Karger AG, Base

Karger' ages, localized to the inner nuclear layer. Based on image distortion of the photoreceptor mosaic, optical modeling suggests that the microcyst had the shape of an aspherical lens, distinguishable from a spherical, cylindrical, or elliptical shape, indicative of an edematous expansion of laminar tissue. The microcyst spontaneously resolved about 30 days after first discovery. No changes to the photoreceptor mosaic ensued from the presence of the microcyst, and functional testing of the photoreceptors below the microcyst indicated no loss of light sensitivity. Conclusions: Microcysts have been associated with numerous subtypes of optic nerve degeneration, including multiple sclerosis and various inherited neuropathies. This microcyst appeared in a healthy individual and resolved without intervention. Lensing effects can be used to determine microcyst shape, which cannot be resolved by OCT imaging, and to help infer etiology.

(c) 2020 S. Karger AG, Basel

\section{Introduction}

Scanning laser ophthalmoscopy (SLO) and optical coherence tomography (OCT) have become essential clinical tools in diagnosing and tracking numerous forms of eye disease and have revealed many details of retinal 
structure in vivo $[1,2]$. One retinal anomaly, described as small (20-90 $\mu \mathrm{m}$ wide) optical gaps in OCT scans, were first described as "retinal pseudocysts" in a retrospective study of geographic atrophy in age-related macular degeneration [3]. These structures are edematous cystoid spaces that lack the epithelial lining of cysts. They are now called microcysts, a pathology that has been associated with a host of diseases [4, 5]. Unlike macular edema, which is detectable on standard en face fundus and fluorescein angiography imaging [6], diagnosis of early microcystic macular edema is generally not visible with these standard fundus imaging techniques. Furthermore, early microcysts have only been diagnosed with OCT and infrared imaging [7].

Typically, microcysts are associated with degenerative retinal diseases. However, a recent report found a microcyst viewed with an adaptive optics SLO (AOSLO) system following trauma from a motor vehicle accident, where the patient was diagnosed with commotio retinae and central vision loss [8]. This microcyst was also seen on an OCT B scan and was located in the inner nuclear layer, similar to the OCT-based findings in diseases. A simple optical model was used in that study to infer a spherical shape for the microcyst based on the photoreceptor density imaged directly beneath the microcyst. Because no images of the photoreceptor mosaic were available before the microcyst appeared, there remains uncertainty regarding the actual shape of this microcyst. Understanding the microcyst's morphology may provide information about its formation.

Here we report the appearance and disappearance of a retinal microcyst, possibly originating from mild head trauma in the month preceding the discovery. Using optical modeling derived from AOSLO images of the photoreceptor layer, we have determined the most likely shape of the microcyst, which appears to be an edematous bulge in a tissue layer rather than a sphere. We also used microstimulation techniques to test whether the microcyst affected light detection during and after the appearance of this transient pathology. Our data provide new insights concerning retinal microcysts that will be useful for disease conditions where microcysts are symptomatic.

\section{Materials and Methods}

\section{Study Patient}

The patient was a 26-year-old Caucasian male with no history of retinal or ocular issues in either eye. He was a participant in ongoing psychophysical research in our laboratory where this study was conducted. Prior to engaging in research imaging, he was screened for ocular abnormalities that would be risk factors for pupil dilation. He had normal visual acuity and color vision. Over the course of a year, the patient was imaged numerous times in the macular area with an AOSLO (described below), and no abnormalities were detected. Early in 2016, the subject experienced a mild head trauma as he collided with the open lid of a commercial trash receptacle. There was no direct impact to the globe or overlying eyelids. Immediately following the incident, the patient noted a headache and general pain on the left side of the face, near the temple, where the collision occurred. The patient did not seek evaluation for concussion, he claimed no lingering symptoms, and he noticed no changes in visual function at any time. One month elapsed before the next AOSLO imaging session, at which time the microcyst was first noticed as an incidental finding. Because of the time frame, we cannot infer any causal relationship between the head trauma and the appearance of the microcyst.

\section{Ocular Imaging}

During the patient's initial screening before unrelated psychophysical experiments, his macula was imaged with spectral domain OCT (SD-OCT; Macular Cube scan, Zeiss Cirrus-4000 OCT). This system has an A-scan rate of $27 \mathrm{kHz}$, imaging with a wavelength of $840 \mathrm{~nm}$ and an optical axial resolution of $5 \mu \mathrm{m}$ in tissue. The OCT scans covered a $6 \times 6 \mathrm{~mm}$ area, with a nominal pixel resolution of $11.7 \mu \mathrm{m}$ in the vertical direction and $46.9 \mu \mathrm{m}$ in the horizontal direction based on the manufacturer's specification. On the same date, he had standard fundus photographs taken (CR-2 Plus AF, Canon, Tokyo, Japan).

AOSLO imaging and microstimulation were performed on an instrument already described extensively [9]; we provide particularly relevant details here. The imaging light source was a diode laser with a center wavelength of $842 \mathrm{~nm}$ and bandwidth of $50 \mathrm{~nm}$ (BLMS-mini, Superlum, Carrigtwohill, Ireland). Adaptive optics correction was performed with a deformable mirror (Multi-DM, Boston Micromachines, Cambridge, MA, USA) positioned to correct the reflected wave front emerging from the eye and traveling through a dual-mirror scanning system operating at a $30 \mathrm{~Hz}$ frame rate. Microstimuli were 1.65 arcmin squares, flashed during 1 video frame, with a wavelength of $543 \mathrm{~nm}$. The exact retinal location of each trial was randomized among 2-3 sites during a session so that accurate relative increment sensitivity was measured during an experiment [10].

To obtain images for one retinal locus, the subject was required to fixate a small target provided by a fiber optic tip illuminated with an amber LED. Head movement was minimized by use of a dental impression bite bar custom fitted for the patient. Upon fixation, 5 to $10 \mathrm{~s}$ videos were acquired using stabilized imaging [11]. We manually removed unstabilized frames (e.g., caused by saccades) before creating an averaged frame for a given focal position. The focal position could be varied by changing the deformable mirror in the AOSLO in $0.01 \mathrm{dpt}$ steps. Averaged images were normalized in contrast and brightness within an 8-bit range for ease of viewing; no other image manipulations were performed. Cone spacing was determined by Fourier transform. All image processing was done with Matlab (Mathworks, Natick, MA, USA).

\section{Optical Modeling and Analysis}

The imaging technologies we used do not have the ability to give detailed information about microcyst shape. AOSLO is diffraction limited and gives high lateral resolution, but its axial reso- 
lution is not sufficient to section the structure in depth. Conversely, the OCT used here can discriminate in depth but is limited in the lateral plane due to poor resolution and pixel sampling. Here we model the propagation of light through simulated microcysts of varying shapes and compare the results to features that are measured from the AOSLO images.

We used the ray-tracing software (Zemax Optical Studio, Zemax, Kirkland, WA, USA) to calculate the point spread functions (PSF) of a scanned imaging beam as it crosses a microcyst. This required creating a standard eye model to focus light at the retina with a single paraxial lens. The eye length was chosen so that the distance from the lens to the external limiting membrane (ELM) was $22.2 \mathrm{~mm}$ with a refractive index of 1.336 [12] and the focal length chosen to get the best focus at the desired depth. The retina was modeled with a distance of $282 \mu \mathrm{m}$ from nerve fiber layer to ELM (measured from the patient's OCT image) with a refractive index of 1.36, taken from the value for the cone photoreceptor myoid and assumed to be typical for transparent retinal tissue [13]. The ELM was used as the 0-depth reference, which we assume is the best focus for photoreceptors, enabling matching of focal depths to AOSLO images. Modeled microcysts were assigned a refractive index of water (1.33).

To simulate the generation of an AOSLO image the amount of light returned to a photodiode when the beam was at radial positions with $1 \mu \mathrm{m}$ spacing relative to the microcyst center were calculated. This was done by importing the wave front, as generated by Zemax using $842 \mathrm{~nm}$ light into Matlab, and the PSF computed by a Fourier transform. The PSF was calculated over a space of 295 $\times 295 \mu \mathrm{m}$ with a resolution of $0.294 \mu \mathrm{m}$. The pupil field amplitude had a Gaussian profile with 1/e full width of $4.5 \mathrm{~mm}$ and was truncated at $5.8 \mathrm{~mm}$ (the beam used in our AOSLO system). As an AOSLO is confocal, the spot falling on a detector is the square of the retinal PSF, and the power is given by its integral. The centroid of the PSF was calculated and its displacement from an ideal beam used to measure distortions. Digitization introduced modulation errors in the model results, so a Loess filter was applied for smoothing. As all modeled microcysts were rotationally invariant with respect to their center, the power and displacement only had to be calculated in a single direction.

We then generated simulated images using predefined reflectance patterns. Two patterns were used, a $10 \times 10 \mu \mathrm{m}$ grid to highlight distortions and a field of Gaussian functions arranged hexagonally with $7 \mu \mathrm{m}$ spacing to mimic cone photoreceptors. These images were generated by calculating where the centroid of a scanned beam lands on the reflectance profile using the modeled displacement, then attenuating this value by the predicted power at the detector. Interpolation was used for both displacement and power values.

Four possible microcyst shapes were modeled, all were centered in depth at $150 \mu \mathrm{m}$ from the nerve fiber layer. The first was a sphere of diameter $56.4 \mu \mathrm{m}$, as measured from the AOSLO images at a depth of $167 \mu \mathrm{m}$ (Fig. 2c). This shape is unlikely, given the axial thickness measured from the OCT images of $40 \mu \mathrm{m}$ but is considered as a reference with the simplest assumptions. The second shape was an ellipsoid defined by

$$
\frac{4 z^{2}}{t^{2}}+\frac{x^{2}+y^{2}}{r^{2}}=1
$$

where $t$ is the axial thickness and $r$ is the lateral radius. The third shape was a cylindrical lens with radius of curvature of both front and back surfaces of $80 \mu \mathrm{m}$. The fourth shape an asphere, with the back surface, $(-t / 2 \geq z \geq 0)$, defined by

$$
\rho=\left(1-q^{3}\right) \rho_{1}+q^{3} \rho_{2}
$$

where $\rho$ is the lateral radius of the shape at a depth $z$, and

$$
q=\frac{z}{t}
$$

weights the summation between

$$
\rho_{1}^{2}=r^{2}\left(1-\frac{z^{2}}{t^{2}}\right)
$$

and

$$
\rho_{2}^{2}=k r^{2}\left(1+\frac{z^{3}}{t^{3}}\right) .
$$

For the asphere, $k=0.7$ was found through optimization. The front surface $(0 \leq z \leq t / 2)$ was simply the mirror image of the back. The first 3 shapes are included as optical components predefined in Zemax. The aspheric shape was created using the freeform $\mathrm{Z}$ function in Zemax, which uses a cubic spline fit using discrete values of $\rho$. We used 21 values of $\rho$ For all shapes, excluding the sphere, $t=40 \mu \mathrm{m}$, as measured from the OCT image, and $r=28.2 \mu \mathrm{m}$, as measured from the image in Figure 1c.

We quantified two values from the AOSLO images of the cones to compare against simulated cone images from the 4 modeled shapes. The first quantity was the change in normalized reflectance intensity with distance from the center of the microcyst. The computed power at the detector was compared to the radial average pixel value using the center of the microcyst as the origin. The second quantity was optical displacement of cones situated beneath the microcyst due to lensing effects. The modeled values of the radial displacement of the focused spot and power at the detector were compared to the measured displacement of cones before and during the microcyst's presence. The latter was determined by identifying the same cones in two such images and plotting the locations of their centers manually.

\section{Statistical Methods}

Root mean square of the residuals was used to quantify the linearly interpolated models versus empirical data for both the optical displacement and intensity results. Goodness of fit of the model data against the empirical data was assessed by the coefficient of determination $\left(R^{2}\right)$. To determine whether certain models gave significantly different results, the residuals were tested using a Wilcoxon rank sum test at the $5 \%$ significance level. Where the simulations were not influenced by the microcyst shapes (i.e., where the beam did not traverse the microcyst), such data were not included in statistical analysis since they all had identical values. All analyses were computed in Matlab.

\section{Results}

During a routine AOSLO imaging session for an ongoing experiment unrelated to this study, a microcyst was discovered by the investigators as the patient searched for the fixation target. The microcyst was located in the right 

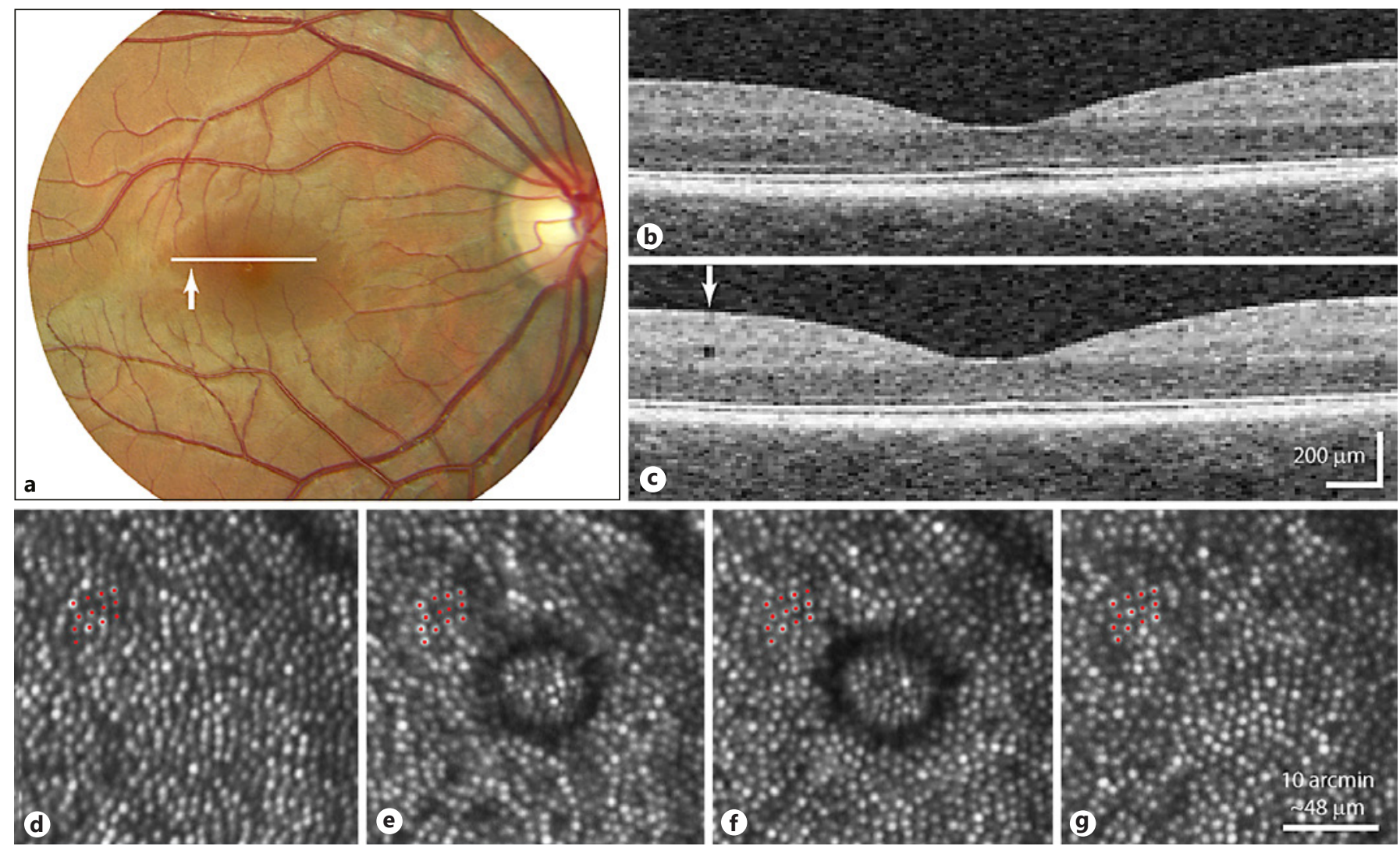

Fig. 1. Visualization of a retinal microcyst with OCT and AOSLO imaging. a A fundus photograph of the patient's right eye. The cross-section of the OCT scans is represented by the white line, while the location of the microcyst is depicted by the white arrowhead at approximately $3.7^{\circ}$ temporal to the fovea. b OCT image through the location depicted in $\mathbf{a}$, taken from a scan acquired over a year before the remaining images were captured. c OCT image through the same location as in $\mathbf{b}$, showing the appearance of the microcyst in the inner nuclear layer 1 day after first discovery. The

field of view of the OCT images has been cropped, rendered with isotropically scaled pixels. Scale dimensions are approximate, based on instrument manufacturer's assumptions about eye dimensions. d An AOSLO image of the area that the cyst appeared in, acquired 13 months earlier. d-g Red dots mark the same 13 cones. e The first appearance of the microcyst with AOSLO. $f$ The last microcyst image, taken 22 days after the first image. $\mathbf{g}$ The microcyst appeared completely resolved here, 29 days after the first image.

eye, $3.7^{\circ}$ from central fixation along the horizontal temporal meridian (Fig. 1a, white arrowhead). The patient was imaged with SD-OCT one day after discovering the microcyst, using the scan pattern that was taken at his initial screening visit, and the cyst was revealed in the SDOCT images (Fig. 1c). The microcyst was present in 3 out of 512 vertically oriented frames and in 1 out of 128 horizontally oriented frames. By detecting the microcyst with these scan dimensions, the SD-OCT findings are in agreement with the size of the microcyst as measured with AOSLO (approx. $56 \mu \mathrm{m}$ in diameter, Fig. 1e, f). The series of images in Figure 1d-g illustrate a consistently undisturbed cone mosaic over time, as indicated with a selection of 13 cones marked in each image. The cone mosaic directly beneath the microcyst was unchanged when

comparing images taken before and after its appearance (Fig. 1d and g; quantified in Fig. 4c), and had cone spacing of 1.33 arcmin which is within the normal range for this eccentricity [14].

Before the microcyst resolved, additional testing was performed. An exploratory search of the central $5^{\circ}$ of the left eye revealed no microcysts or apparent changes to the photoreceptor layer. Also, the patient performed psychophysical testing of the photoreceptor mosaic, with microstimuli targeted outside of, on the border of, and within the center of the microcyst. In each location, the patient was able to detect light at threshold levels consistent with normal subjects tested previously on the same equipment [10]. These results indicate that the presence of the microcyst did not interfere with light detection. 

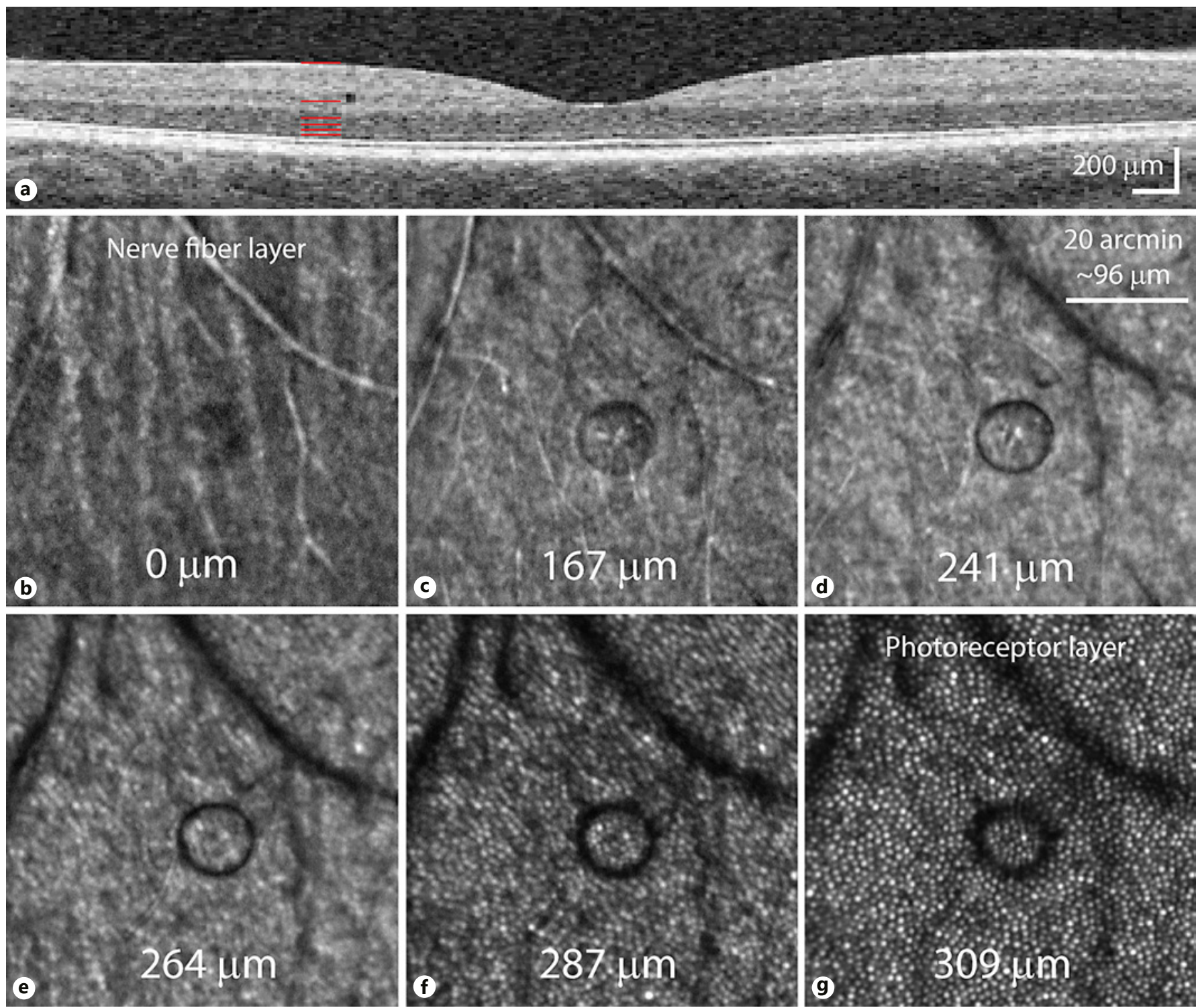

Fig. 2. Microcyst appearance in different focal planes. a OCT scan taken 1 day after microcyst discovery, showing its location in the inner nuclear layer. Pixels are scaled to be isotropic. Red lines depict the corresponding depths of the AOSLO images in $\mathbf{b}-\mathbf{g}$, with depths estimated from the defocus used during capture (photoreceptors appeared sharpest at one depth, assumed to be near the ELM, that was calculated to be $309 \mu \mathrm{m}$ below the nerve fiber layer

image). b The nerve fiber layer shows a hyporeflective microcyst area. The microcyst border appears distinctly at $167 \mu \mathrm{m}$ depth (c), becoming darker at $241 \mu \mathrm{m}$ (d). As the imaging plane moves toward the photoreceptor layers (e-g), the microcyst becomes less well-defined and darker still, as it deflects more light away from the AOSLO light collection path.

In addition to perceptual testing, a series of images were collected in different focal planes of the retina (Fig. 2). The microcyst is just visible at the plane of the nerve fiber layer (Fig. 2b), and it changes appearance as the imaging plane is moved deeper into the retina. The sharpest focus of the microcyst border occurs around 68 $\mu \mathrm{m}$ from the ELM (Fig. 2d), and the border becomes in-

creasingly less defined as the focus is moved toward the photoreceptor layer (Fig. 2g). The microcyst was monitored with AOSLO imaging approximately once a week for almost 4 weeks (Fig. 1d-g). Sometime between 21 and 28 days after its discovery, the microcyst spontaneously resolved. After the disappearance, the patient was examined by an ophthalmologist, including fundus photogra- 


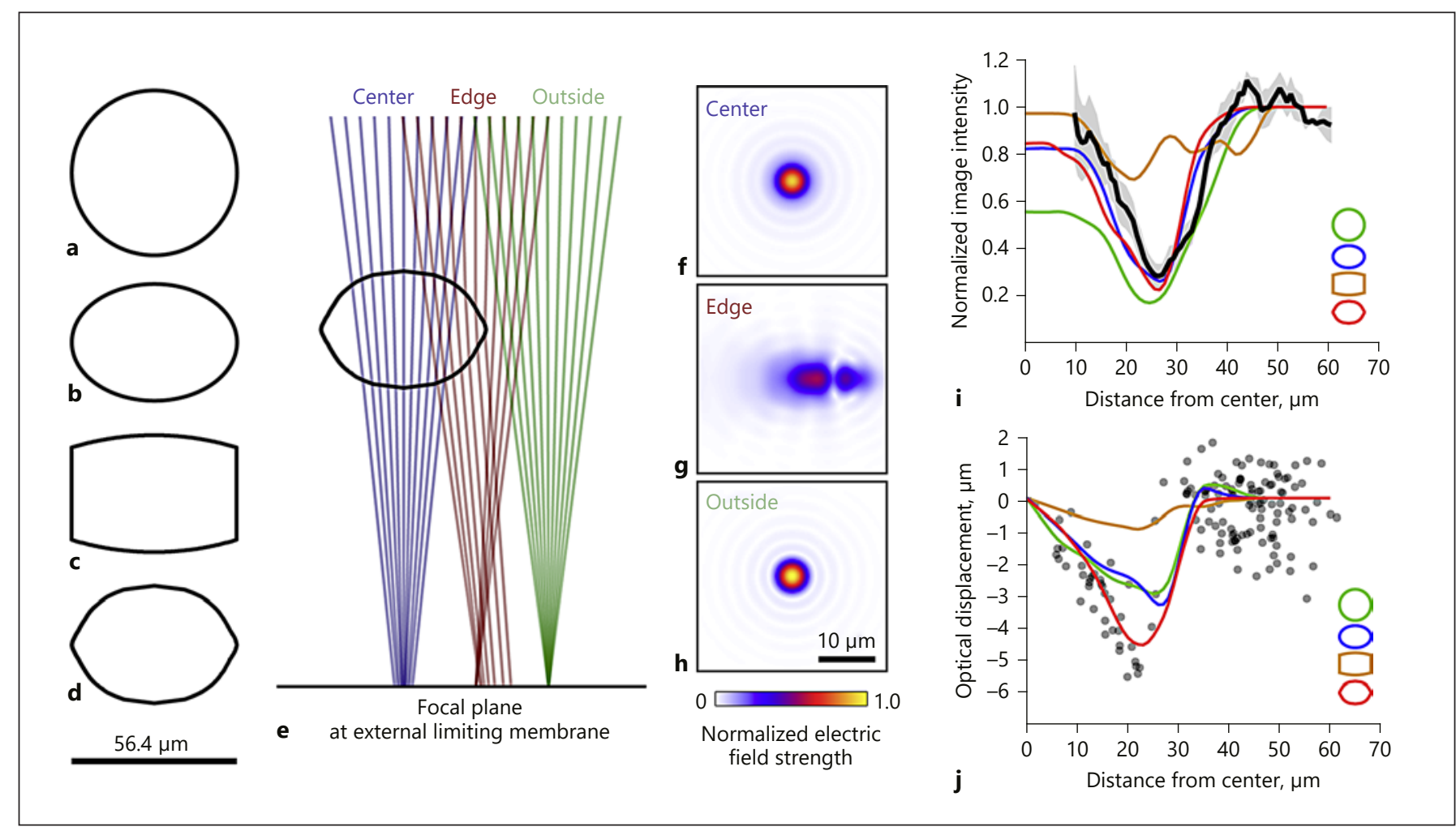

Fig. 3. Optical modeling of microcyst shapes. Four simulated shapes were used to estimate the morphology of the microcyst: spherical (a), elliptical (b), cylindrical (c), and aspheric (d). e Example ray traces passing through the aspheric shape at the center (blue), edge (red), and outside of the microcyst (green). f-h Point spread functions (PSF) corresponding to the traces shown in e, depicted as the modulus of the electric fields and normalized to the peak value found in $\mathbf{h}$. i Average radial intensity measured from the center of the microcyst (black line, \pm 1 SEM in gray, from Fig. 1f) compared to radial intensities for the simulated shapes (colored lines matching shapes in legend). j Optical displacements induced by simulated shapes of the PSF centroid (colored lines) compared to measured displacements for cones located inside and outside the microcyst (gray points; see Fig. 4d). phy and slit lamp assessment in both eyes, which yielded no remarkable findings.

We then used the AOSLO imaging data to estimate what the 3-dimensional shape of the microcyst would be, given the patent lensing effects. By simulating a scanned beam across various microcyst shapes (Fig. $3 a-d$ ) we could calculate the expected degree of shadowing of the cone mosaic as well as the radial displacement of the cone positions. When the focus was positioned at the ELM, there were clear differences between the simulated shapes. For all shapes, when the beam is at the center of the microcyst the curvature causes some defocus (e.g., blue lines in Fig. 3e). The focal point is shifted outwards, past the photoreceptors due to the microcyst's lower refractive index, which leads to dimming as seen in the AOSLO images. As the beam moves towards the microcyst edge, the cone of light is distorted by the lateral step in refractive index between microcyst and surrounding tissue. Consequently, the beam is only partially focused at the image plane, lowering the resolution, the intensity in the image, and thereby creating a shadow. These optical consequences are best characterized by the PSF for each beam position (Fig. $3 \mathrm{f}-\mathrm{h}$ ) which show distortion and dimming.

The radial intensity of the modeled shadows shows that only the ellipse and aspheric shapes give a reasonable fit to the AOSLO imaging data (Fig. 3i). The root mean square residuals of the cylindrical, spherical, elliptical, and aspheric shapes to the measured data were $0.28,0.22,0.11$, and 0.14 , while the $\mathrm{R}^{2}$ values were -0.23 , $0.25,0.82$, and 0.68 , respectively. The aspherical and elliptical shapes have predictions that closely match the imaging data but are not significantly different from each other $(p=0.61)$. When considering the optical distortion (Fig. 3j), all modeled shapes yield two phases; increased displacement up to the boundary of the cyst, 

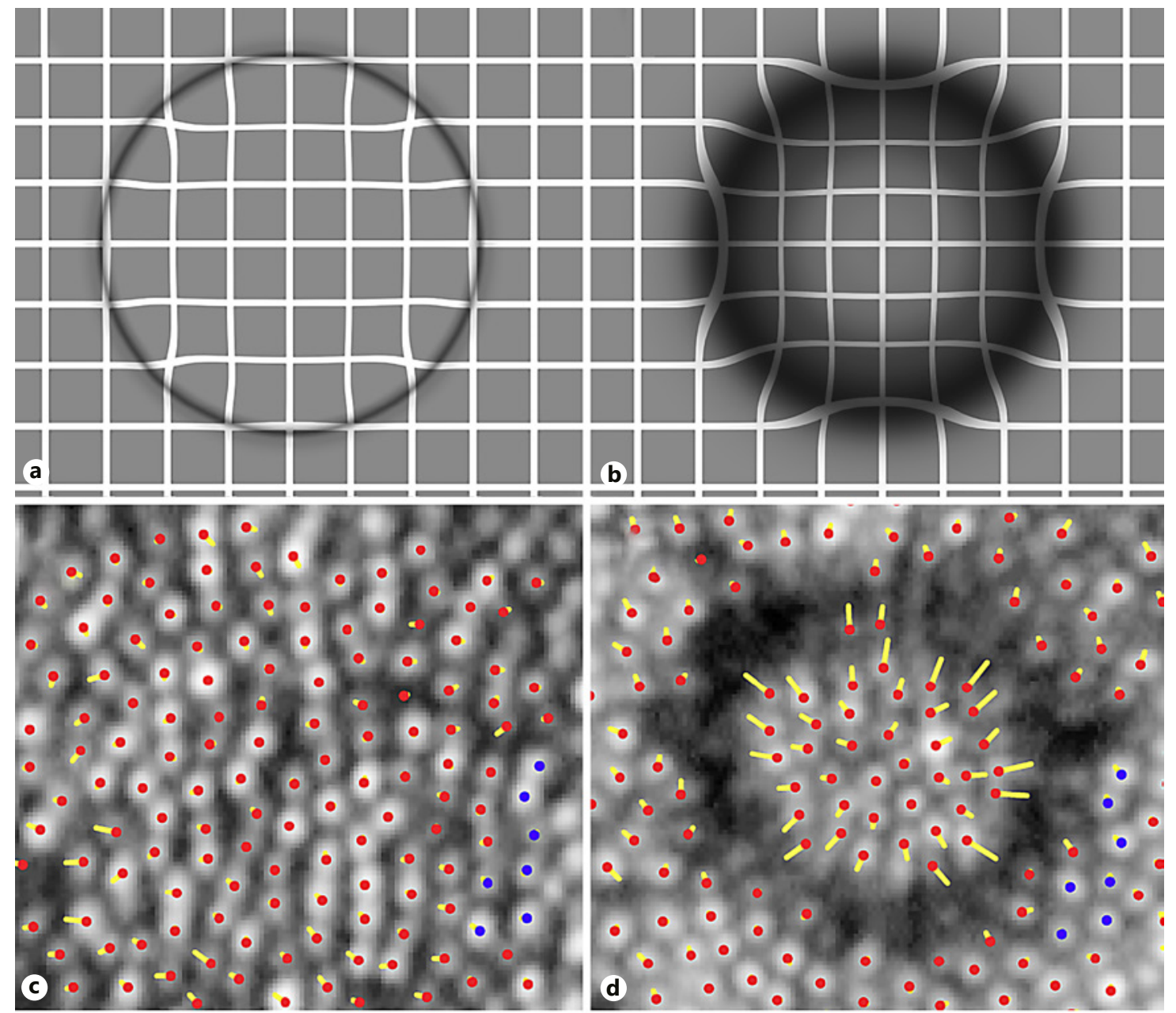

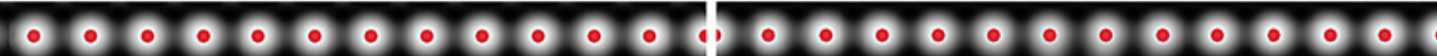
$00000000000000.1,1,0000$ $0000000000.00011,1,1,000$

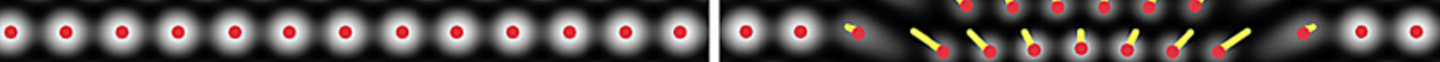

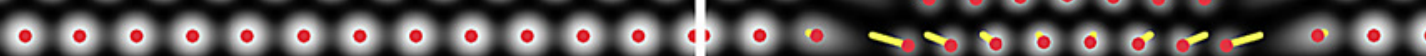

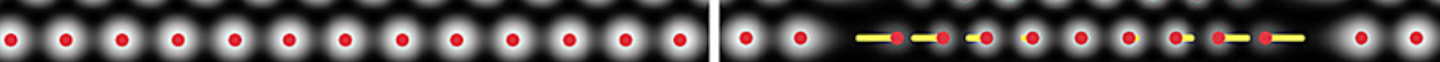

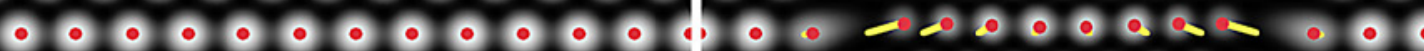

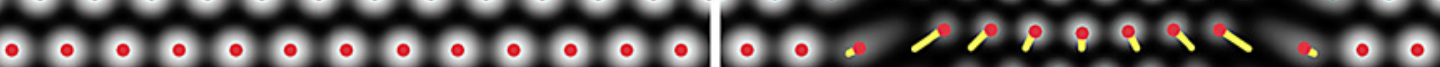

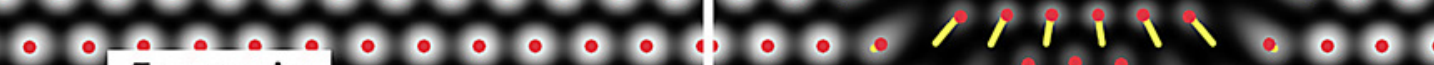
e. $5 \operatorname{arcmin} 54 \mu \mathrm{m}$

Fig. 4. Microcyst aspheric model compared to AOSLO cone image. a Simulated image obtained at a focal plane just below the aspheric shape model, which compares well with the AOSLO image in Figure 2c. b Simulated image with the focus set at the photoreceptor layer, showing diffuse hyporeflectivity and substantial optical distortion. Log scaled AOSLO images of the microcyst taken before (c) and after its appearance $(\mathbf{d})$. Red dots indicate the cone centers, and yellow lines show their displacement vectors based on cone locations imaged after the microcyst resolved. Blue dots indicate the same set of 7 cones in both images, showing that the same patch of cone mosaic is depicted. The data in c demonstrate that no cones were lost during the microcyst's existence. Simulated cone array with spacing of $7 \mu \mathrm{m}(\mathbf{e})$ used to compute optical displacement due to an aspheric microcyst (f). Red dots are cone centers and yellow lines show displacement vectors. 
then a sharp transition to a flat regime, even before the whole beam has exited the cyst, with the lens shape showing the least distortion. The root mean square residuals of the cylindrical, spherical, elliptical, and aspheric shapes to the measured data were $1.83,1.24$, 1.29 , and 1.08 , respectively. The $\mathrm{R}^{2}$ values were -0.03 , $0.53,0.48$, and 0.64 (in the same order). For values $<45$ $\mu \mathrm{m}$, residuals between spherical and elliptical models showed no significant difference $(p=0.93)$, but both were significantly different from the aspheric shape $(p=$ 0.007 and 0.0098 , respectively). These modeling results suggest that the aspheric shape best captures the morphological features of the microcyst.

To confirm these findings, we compared the pattern of distortions seen in AOSLO and simulated aspheric images (Fig. 4a, b). In particular, the apparent movement of photoreceptors - both real and modeled - show predictable shifts in position (Fig. 4c-f). The variable displacement of the focal spot with respect to radial distance from the center also causes the photoreceptors in the shadowed area to be appreciably distorted.

\section{Discussion}

Why retinal microcysts develop - either in disease or from trauma - is unclear, and multiple mechanisms are suspected [5, 15]. Microcysts have not been reported in any healthy controls in other studies ( 289 healthy controls in 4 studies) [4,16-18]. The variability of OCT scan patterns used in clinical imaging would limit their detection, as these structures are likely only seen with consecutive scans that are less than half the diameter of the average microcyst. With lower scan density, these microcysts will only be visualized by chance, and may appear as shadowing artifacts if the scan is not directly through the center of the structure.

It is not uncommon for microcysts to resolve. A longitudinal study of 15 multiple sclerosis patients found that 6 had dynamic changes over 3-12 months, with microcysts resolving in 2 patients [16]. In glaucoma, microcysts grew in 2 of 13 eyes studied, with some becoming indistinct in OCT over many months [17]. Given the widespread occurrence of microcysts across numerous optic neuropathies, and the finding that microcysts are most prevalent in patients with mitochondrial hereditary optic neuropathies, it has been proposed that they arise from mitochondrial dysfunction, possibly in Müller cells [19]. The other AOSLO report of a microcyst structure implies that the trauma suffered by the patient could have led to the formation of the structure [8]. We noted that the patient in this study did have a mild head injury about 1 month prior to the discovery of the microcyst, although he reported no visual or cognitive symptoms associated with the incident. At present, the cause of these traumainduced microcystioid structures remains unknown, although their location in the inner nuclear layer suggests some susceptibility for laminar shearing or schisis at the soma/synapse boundary.

The microcyst did not appear to affect visual detection of stimuli delivered to the cone mosaic directly beneath the cyst, whether in the center or the shadowed ring. However, given the displacement of ganglion cells within the central $15^{\circ}$ of the retina, it is possible that the affected visual region may have actually been closer to the fovea [20]. If there was synaptic disruption or ganglion cell injury associated with the microcyst, the affected cells would have probably served a visual area located $0.8-1.2^{\circ}$ from the cyst, towards the fovea [21]. In the present study, the microcyst resolved before we could explore other areas for visual deficits.

The distortion and shadowing are caused by the curvature of the microcyst's structure and lateral changes in refractive index encountered by the incident beam. Lateral step changes like this are a known issue in microscopy [22], reducing brightness and resolution. If microcysts are present in a retina, this will affect quantification of cone mosaics in disease. For example, the distortion caused by cysts may give an inaccurate photoreceptor density measurement below it, or shadowing may suggest hyporeflectivity in the cones, where there is none. Such effects are evident in well-documented cases of commotio retinae [23].

We cannot be certain that the modeling reveals the correct shape, as there may be untested shapes that produce better fits to the data, but using the information from the models we can determine likely features. When a scanned beam passes through the center of a microcyst, the beam will be defocused to a degree proportional to the radius of curvature, and photoreceptor reflectivity will be inversely related to this defocus. Consequently, shapes with high curvature - such as a sphere - will lead to dim cones, which is not what is observed in this patient (Fig. 3i, $<20 \mu \mathrm{m}$ ). Likewise, the apparent displacement of cones will be sensitive to the steepness of the microcyst edges (Fig. 3j). Because lensing effects are optically predictable from images of the cone mosaic, alternative microcyst shapes that may occur in different patients or at different time points could be determined from similar analyses. Our results only apply to this specific micro- 
cyst, imaged at unknown stages in its life cycle. If microcysts form and then disappear, it is likely that differences in shape will occur during its life span. Cysts with longer life spans, such as those seen in retinal diseases, often develop into more elaborate, multilobed shapes. Nonetheless, our results show how modeling of such structures can provide more information than imaging alone, potentially offering clues for how microcysts form and develop.

\section{Statement of Ethics}

All data collection and experiments were performed under an approved Institutional Review Board protocol through the University of Alabama at Birmingham and adhered to the tenets of the Declaration of Helsinki. The patient was informed of the risks involved and gave written consent prior to beginning the study.

\section{Disclosure Statement}

A.M.: none. A.S.M.: at the time of data collection and analysis, none; at the time of submission, this author was employed by Novartis Pharmaceutical Company. B.C.S.: Heidelberg Engineering Inc. Research Support. L.C.S.: none.

\section{Funding Sources}

NIH grants R01-EY023581, R01-EY023591, P30-EY003039, and the Eyesight Foundation of Alabama.

\section{Author Contributions}

A.M., A.S.M, and L.C.S. collected and analyzed the imaging data. B.C.S. performed the ophthalmic exam and assessed OCT images. A.M. created the optical model. A.M. and A.S.M. wrote the first draft of the manuscript. All authors edited the paper into its final form.

\section{References}

1 Puliafito CA, Hee MR, Lin CP, Reichel E, Schuman JS, Duker JS, et al. Imaging of macular diseases with optical coherence tomography. Ophthalmology. 1995 Feb;102(2):21729.

2 van Velthoven ME, Faber DJ, Verbraak FD, van Leeuwen TG, de Smet MD. Recent developments in optical coherence tomography for imaging the retina. Prog Retin Eye Res. 2007 Jan;26(1):57-77.

3 Cohen SY, Dubois L, Nghiem-Buffet S, Ayrault S, Fajnkuchen F, Guiberteau B, et al. Retinal pseudocysts in age-related geographic atrophy. Am J Ophthalmol. 2010 Aug;150(2): 211-217.e1.

4 Burggraaff MC, Trieu J, de Vries-Knoppert WA, Balk L, Petzold A. The clinical spectrum of microcystic macular edema. Invest Ophthalmol Vis Sci. 2014 Feb;55(2):952-61.

5 Bhargava P, Calabresi PA. The expanding spectrum of aetiologies causing retinal microcystic macular change. Brain. 2013 Nov; 136(Pt 11):3212-4.

6 Fardeau C, Champion E, Massamba N, LeHoang P. Uveitic macular edema. Eye (Lond). 2016 Oct;30(10):1277-92.

7 Abegg M, Dysli M, Wolf S, Kowal J, Dufour P, Zinkernagel M. Microcystic macular edema: retrograde maculopathy caused by optic neuropathy. Ophthalmology. 2014 Jan;121(1): 142-9.

8 Langlo CS, Flatter JA, Dubra A, Wirostko WJ, Carroll J. A lensing effect of inner retinal cysts on images of the photoreceptor mosaic. Retina. $2014 \mathrm{Feb} ; 34(2): 421-2$.
9 Harmening WM, Tuten WS, Roorda A, Sincich LC. Mapping the perceptual grain of the human retina. J Neurosci. 2014 Apr;34(16): 5667-77.

10 Bruce KS, Harmening WM, Langston BR, Tuten WS, Roorda A, Sincich LC. Normal perceptual sensitivity arising from weakly reflective cone photoreceptors. Invest Ophthalmol Vis Sci. 2015 Jul;56(8):4431-8.

11 Yang Q, Arathorn DW, Tiruveedhula P, Vogel CR, Roorda A. Design of an integrated hardware interface for AOSLO image capture and cone-targeted stimulus delivery. Opt Express. 2010;18(17):3283-91.

12 Atchison DA, Thibos LN. Optical models of the human eye. Clin Exp Optom. 2016 Mar; 99(2):99-106

13 Spaide RF, Curcio CA. Anatomical correlates to the bands seen in the outer retina by optical coherence tomography: literature review and model. Retina. 2011 Sep;31(8):1609-19.

14 Chen Y, Ratnam K, Sundquist SM, Lujan B, Ayyagari R, Gudiseva VH, et al. Cone photoreceptor abnormalities correlate with vision loss in patients with Stargardt disease. Invest Ophthalmol Vis Sci. 2011 May;52(6):328192.

15 Sigler EJ. Microcysts in the inner nuclear layer, a nonspecific SD-OCT sign of cystoid macular edema. Invest Ophthalmol Vis Sci. 2014 May;55(5):3282-4

16 Gelfand JM, Nolan R, Schwartz DM, Graves J, Green AJ, Gelfand JM, et al. Microcystic macular oedema in multiple sclerosis is associated with disease severity. Brain. 2012 Jun;135(Pt 6):1786-93.
17 Hasegawa T, Akagi T, Yoshikawa M, Suda K, Yamada H, Kimura Y, et al. Microcystic inner nuclear layer changes and retinal nerve fiber layer defects in eyes with glaucoma. PLoS One. 2015 Jun;10(6):e0130175.

18 Saidha S, Sotirchos ES, Ibrahim MA Crainiceanu CM, Gelfand JM, Sepah YJ, et al. Microcystic macular oedema, thickness of the inner nuclear layer of the retina, and disease characteristics in multiple sclerosis: a retrospective study. Lancet Neurol. 2012 Nov; 11(11):963-72.

19 Wolff B, Azar G, Vasseur V, Sahel JA, Vignal $C$, Mauget-Faÿsse M. Microcystic changes in the retinal internal nuclear layer associated with optic atrophy: a prospective study. J Ophthalmol. 2014;2014:395189.

20 Curcio CA, Allen KA. Topography of ganglion cells in human retina. J Comp Neurol. 1990 Oct;300(1):5-25.

21 Watson $A B$. A formula for human retinal ganglion cell receptive field density as a function of visual field location. J Vis. 2014 Jun; 14(7): 15 .

22 Jonášová EP, Bjørkøy A, Stokke BT. Recovering fluorophore concentration profiles from confocal images near lateral refractive index step changes. J Biomed Opt. 2016 Dec;21(12): 126014

23 Braza ME, Young J, Hammeke TA, Robison SE, Han DP, Warren CC, et al. Assessing photoreceptor structure in patients with traumatic head injury. BMJ Open Ophthalmol. 2018 Nov;3(1):e000104. 